\title{
The measurement of temporal stream of hearing by continuous judgments - In the case of the evaluation of helicopter noise
}

\author{
Seiichiro Namba,* Sonoko Kuwano,* and Masaru Koyasu** \\ *College of General Education, Osaka University, \\ 1-1 Machikaneyama, Toyonaka, Osaka, 560 Japan \\ **Acoustical Engineering Laboratory, \\ 2-13-11-806 Shinjuku, Shinjuku-ku, Tokyo, 160, Japan
}

(Received 30 October 1992)

\begin{abstract}
In our daily lives we are surrounded with a variety of sounds which vary with time and spread in space. The configurations of sound along the temporal stream have the meaning. The meaning will be destroyed if these sounds are divided into short segments as used in conventional methods. The method of continuous judgment by category and the method of continuous judgment by selected description have been developed for examining the impression of temporally varying sounds along the temporal stream. In this paper these methods are introduced with their procedures and applications to the evaluation of helicopter noise.
\end{abstract}

Keywords: Continuous judgment, Helicopter noise

PACS number: 43. 50. Qp, 43. 50. $-\mathrm{x}$

\section{INTRODUCTION}

In our daily lives we are surrounded with a variety of stimuli which vary with time and spread in space. The configurations of sounds along the temporal stream have the meaning. The meaning will be destroyed if these sounds are divided into short segments as used in conventional methods. It is desirable to examine the impression of temporally varying sounds along the stream.

The authors have developed new methods called "the method of continuous judgment by category"1-3) and the method of selected description ${ }^{4-6)}$ in order to evaluate continuously the impressions of sounds which vary with time.

The idea of these new methods was created by Namba, who thought that the impressions of musical performances can be expressed by the movement of fingers since piano performances are played with fingers. It is not unusual that some passages in piano music are played faster than the critical rate for identification. ${ }^{7)}$ This fact suggests that it would be possible to measure the impression of fluctuating sounds continuously by pressing a key on a response box as quickly as critical rate, as is done in the piano training.

Music has a Gestalt including all the sounds already presented and coming in future, though each sound appears and disappears one after the other. The instantaneous impression of the musical sound is based not only on each sound presented at that moment, but on the whole musical context. This holds good for speech or other sounds. It can be easily understood that the instantaneous impressions of speech sounds are greatly dependent on the context.

Subjects are asked to judge not only the instantaneous impressions, but the overall impression of the sound, and the relation between instantaneous judgments and overall judgments are examined. 
This may be helpful to understand the interaction between the instantaneous impressions and overall impressions. Instantaneous impressions exist in the reference frame of whole stimulus and the overall impression is largely dependent on the instantaneous impressions. The method of continuous judgment by category and the method of continuous judgment by selected description are not effective to be applied to sounds without frame of reference, e.g. artificial sounds without meaning.

In the experimental design using conventional psychological methods, the stimuli are presented in random order in order to avoid constant errors. It can be easily understood that it is useless to apply the methods of continuous judgment by category and by selected description to such randomly presented short segments of stimuli.

The methods of continuous judgment can be applied to sounds which have a meaningful context and whose instantaneous impressions exist along the temporal stream reflecting the context, such as music, speech and sounds from noise sources in our daily life. Music and speech have context. Also noise sources have context when they are moving such as transportation noises. From the context we can perceive from which direction the sound source is coming, predict to which direction it is moving, and we can smoothly react to the instantaneous condition of the sound source.

The helicopter noise is a typical example of this kind of noise, since the level and timbre of helicopter noise drastically varies with time according to the distance between helicopters and listeners. In order to evaluate helicopter noise, it is necessary to measure instantaneous impressions of the noise according to the flight pattern, and then examine the relation between instantaneous and overall impressions.

In the present study, the method of continuous judgment by category and the method of continuous judgment by selected description will be introduced and the measurement of temporal stream of hearing will be discussed by taking the evaluation of helicopter noise as an example.

\section{EXPERIMENTS}

\subsection{Stimuli}

A list of the helicopter noise used in experiments is shown in Table 1. They were in three flying mode; taking-off, flyover and landing. Stimuli No. 1 to 4 in Table 1 have no frequency equalization, stimuli No. 5 to 21 were equalized with an frequency equalizer (Victor SEA-7070) so that the frequency characteristics of white noise might be as flat as possible when presented through the loudspeakers in the sound proof room, and stimuli No. 22 to 25 were equalized as if they were transmitted through facades having idealized sound transmission characteristics $(-3 \mathrm{~dB} / \mathrm{oct})$. These stimuli were modified using a mixer to creat a panning effect so that it might seem that the helicopter was moving in space. In experiment these stimuli were reproduced with a DAT recorder (Victor XD-Z1100) and presented to subjects through an amplifier (Technics SU-V100D) and two loudspeakers (Sony APM-33W) which are mounted on the ceiling of the sound proof room.

\subsection{Procedure: Experiment 1-By the Method of Continuous Judgment by Category}

\section{(1) Instruction}

The instantaneous impression of noisiness was judged using the method of continuous judgment by category. The instruction given to subjects is shown below. The outline of the method of continuous judgment by category will be understood by this instruction.

"This is an experiment on the noisiness of helicopter noise. Various helicopter noises will be presented to you from the loudspeakers. The noisiness of the sounds will vary with time. Please judge the noisiness at each moment using 7 categories from "intolerably noisy" to "not noisy at all" by touching the corresponding key. You need not press the key if your impression of noisiness does not change. The impression on the monitor will remain. When your impression of noisiness changes, pleases press the corresponding key."

\section{(2) Response box}

It is necessary to store the response of subjects with the time when they respond. For that purpose, a computer keyboard is used as a response box.

\section{(3) Number of categories}

In our experiments using the method of continuous judgment by category, seven categories are usually used. In our earlier experiment ${ }^{8)}$ using 5 to 11 categories, there was no significant effect of the number of categories on the judgments. However, there is a case that subjects feel like to express a slight change of their impression which is too slight to use a different category. For such cases, it would 


\section{S. NAMBA et al.: MEASUREMENT OF TEMPORAL STREAM OF HEARING}

Table 1 Stimulus conditions.

\begin{tabular}{rcccccc}
\hline No. & $\begin{array}{c}\text { Flight } \\
\text { mode }\end{array}$ & $\begin{array}{c}\text { Number of } \\
\text { blades }\end{array}$ & Equalizing & $\begin{array}{c}\text { Altitude } \\
\text { (ft) }\end{array}$ & $\begin{array}{c}L_{\text {eq }} \\
(\mathrm{dBA})\end{array}$ & $\begin{array}{c}\text { Duration } \\
\text { (s) }\end{array}$ \\
\hline 1 & L/D & 2 & - & $1,000 \rightarrow 458$ & 81.3 & 61.0 \\
2 & L/D & 2 & - & $1,500 \rightarrow 528$ & 82.5 & 57.4 \\
3 & L/D & 5 & - & $1,500 \rightarrow 828$ & 72.3 & 41.5 \\
4 & L/D & 2 & - & $1,000 \rightarrow 438$ & 82.1 & 58.4 \\
5 & T/O & 2 & flat & $395 \rightarrow 1,000$ & 73.4 & 56.6 \\
6 & T/O & 5 & flat & $315 \rightarrow 1,000$ & 68.3 & 45.0 \\
7 & T/O & 5 & flat & $465 \rightarrow 1,500$ & 68.0 & 54.4 \\
8 & T/O & 3 & flat & $315 \rightarrow 1,500$ & 67.4 & 51.2 \\
9 & T/O & 2 & flat & $325 \rightarrow 1,000$ & 77.4 & 44.9 \\
10 & T/O & 2 & flat & $555 \rightarrow 1,000$ & 75.3 & 50.4 \\
11 & L/D & 2 & flat & $1,000 \rightarrow 458$ & 78.9 & 60.4 \\
12 & L/D & 2 & flat & $1,500 \rightarrow 528$ & 80.2 & 57.1 \\
13 & L/D & 5 & flat & $1,500 \rightarrow 828$ & 70.0 & 41.3 \\
14 & L/D & 2 & flat & $1,000 \rightarrow 438$ & 80.1 & 58.2 \\
15 & L/F & 2 & flat & 925 & 72.9 & 68.6 \\
16 & L/F & 5 & flat & 925 & 65.5 & 46.9 \\
17 & L/F & 5 & flat & 1,425 & 63.3 & 57.4 \\
18 & L/F & 3 & flat & 925 & 61.8 & 64.1 \\
19 & L/F & 3 & flat & 1,425 & 58.5 & 52.4 \\
20 & L/F & 2 & flat & 925 & 73.8 & 57.3 \\
21 & L/F & 2 & flat & 1,425 & 70.9 & 55.5 \\
22 & L/D & 2 & -3 dB/oct. & $1,000 \rightarrow 458$ & 76.2 & 58.9 \\
23 & L/D & 2 & -3 dB/oct. & $1,500 \rightarrow 528$ & 77.4 & 53.5 \\
24 & L/D & 5 & -3 dB/oct. & $1,500 \rightarrow 828$ & 65.9 & 37.2 \\
25 & L/D & 2 & -3 dB/oct. & $1,000 \rightarrow 438$ & 77.3 & 52.6 \\
\hline
\end{tabular}

be suitable to use response methods which vary continuously. One of such methods is a crossmodality matching between an auditory attribute and the length of a line. Scharf has proposed successive magnitude estimation, ${ }^{9)}$ but the use of numerals is not appropriate to the rapid change of impressions. Instead of using numerals, the length of a line is used in our revised successive magnitude estimation. ${ }^{10,11)}$ Subjects are asked to adjust the length of a line on a computer display so that the length may correspond to the impression of sounds. In order to avoid the limitation of the length, it is desirable to project the line on a large screen.

(4) Estimation of reaction time

There is a time lag (reaction time) between the presentation of sounds and the responses of subjects to them. The reaction time can be estimated from the coefficient of correlation between physical values and subjects' responses. An example of the relation between the coefficient of correlation and the time lag is shown in Fig. 1.3) The time lag when the highest correlation can be obtained is regarded as the reaction time.
2.3 Procedure: Experiment 2-by the method of continuous judgment by selected description

\section{(1) Method}

The method of continuous judgment by category can only be used for measuring responses in one dimension such as loudness or noisiness. The impression of sounds such as music or helicopter noise is multidimensional and temporally varying. In order to measure the instantaneous multidimensional impression continuously, the method of continuous judgment by selected description has been developed..$^{4-6)}$ Basically this method is similar to the method of continuous judgment by category. In the experiment using the method of selected description subjects are asked to judge the instantaneous impression of timbre of sounds using adjectives in a prepared list and press the corresponding key. When they feel two or three descriptions are appropriate, they can use two or three keys in quick succession, like playing a trill or a broken chord on a piano. In Experiment 2 the instantaneous impression of timbre of helicopter noise was measured continuously using the method of continuous judg- 
ment by selected description.

(2) List of adjectives

A list of adjectives is prepared on the basis of the

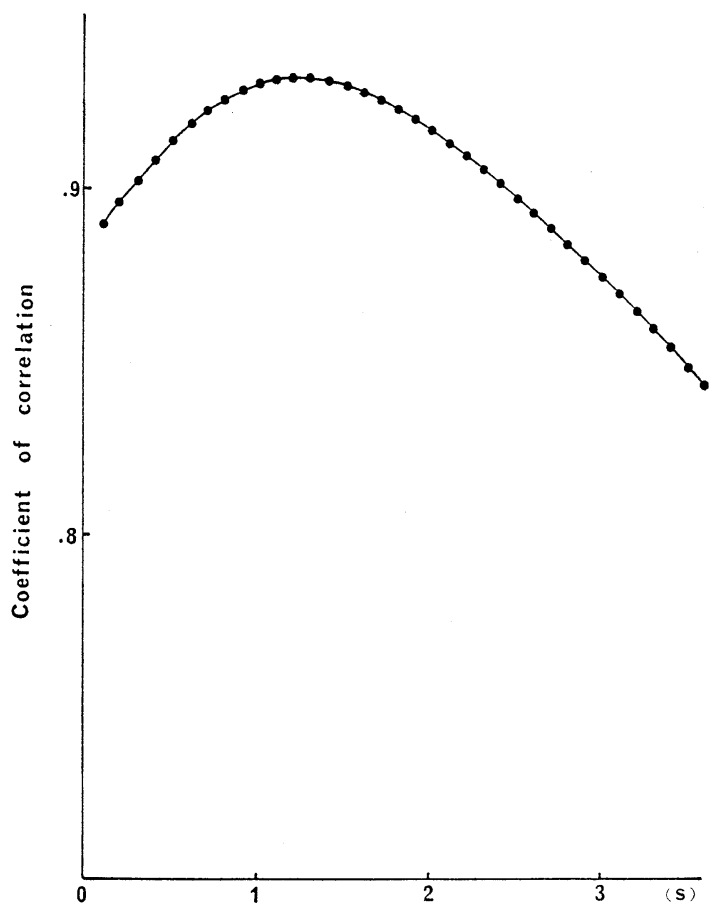

Fig. 1 Relation between coefficient of correlation and time interval. ${ }^{3)}$ A systematic change in the coefficient of correlation was found in accordance with the time interval between sound levels and the instantaneous judgments. results of preliminary experiments. In the first preliminary experiment, subjects are asked to express their impression to each sound using any adjectives they like. In the second preliminary experiment subjects are asked to select adjectives appropriate to express the impression of each sound from the list of adjectives which is prepared on the basis of the results of the first preliminary experiment. Cluster analysis can be conducted on the basis of the frequency with which the adjectives are commonly selected. An example is shown in Fig. 2. ${ }^{12)}$

The list of adjectives used in Experiment 2 is shown in Table 2 with their symbol letters, the key of which subjects were asked to press. These symbol letters were determined so that subjects could easily associate them with the adjectives. For example, "Y" is the first letter of "yakamashii" (which means "noisy" in Japanese). It can be noticed that these adjectives are the representatives of each cluster shown in Fig. 2. Subjects are required to have a training sufficient to get used to the keys with cor-

Table 2 List of adjectives used in Experiment 2 .

\begin{tabular}{cll}
\hline Symbol & \multicolumn{2}{c}{ Adjective } \\
\hline $\mathrm{Y}$ & noisy & $($ やかましい) \\
$\mathrm{C}$ & soft & $($ 小さい) \\
$\mathrm{H}$ & powerful & (迫力のある) \\
$\mathrm{M}$ & metallic & (金属性の) $)$ \\
$\mathrm{N}$ & dull & $($ にぶい) \\
$\mathrm{K}$ & hard & $($ かたい)
\end{tabular}

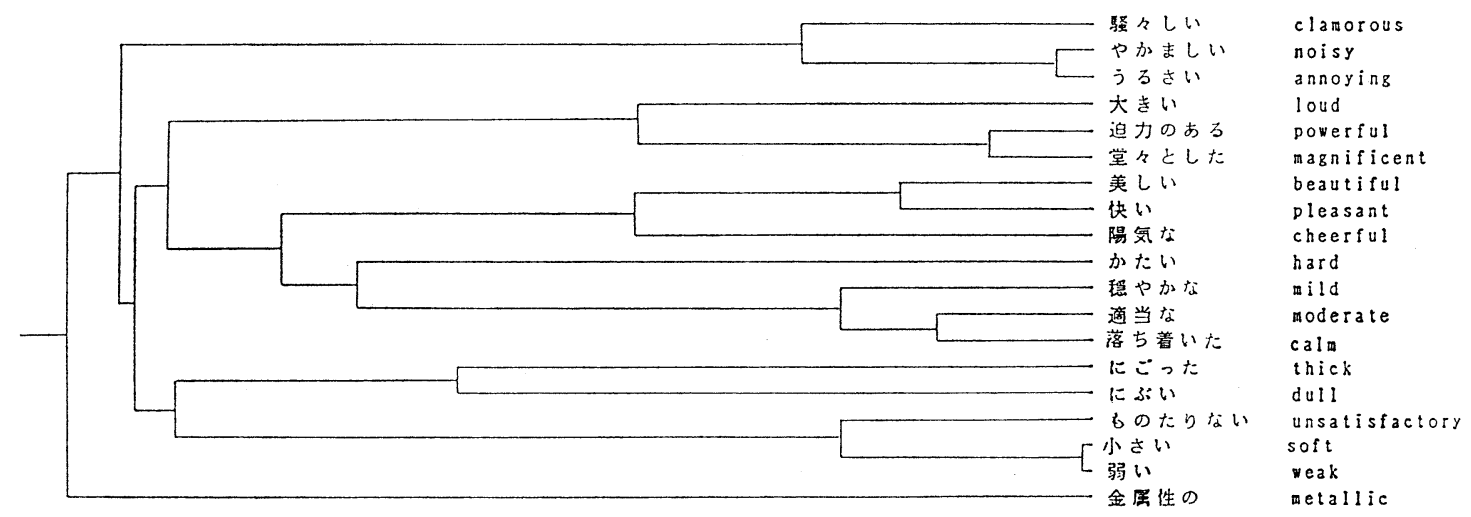

Fig. 2 The results of cluster analysis for adjectives in the experiment using the method of selected description. ${ }^{12)}$ 
responding adjectives before experiments.

\subsection{Procedure: Experiment 3-Overall Judgment Using Semantic Differential}

Helicopter noises produce various impression of timbre. Therefore the overall impression of the helicopter noises were multidimensionally examined using semantic differential. The adjective scales used are shown in Table 4. Subjects were asked to judge their impression of timbre using these 7-point adjective scales after listening to each stimulus.

Experiments 1, 2 and 3 were conducted in different days in different order among subjects.

\subsection{Subjects}

Five females and seven males, aged between 20 and 41 , with normal hearing ability participated in the experiments as subject.

\section{RESULTS}

\subsection{Instantaneous Impression of Noisiness}

The continuous judgments of each subject to each stimulus were sampled every $100 \mathrm{~ms}$ and corresponded to the values of $L_{\text {eq }}$ of every $100 \mathrm{~ms}$. The instantaneous judgments by twelve subjects were averaged taking the reaction time into account. The coefficients of correlation between instantaneous judgments sampled every $100 \mathrm{~ms}$ and the values of $L_{\text {eq }}$ of every $100 \mathrm{~ms}$ for each stimulus is shown in Table 3. High correlation can be seen between them for all the 25 stimuli. This suggests that the instantaneous impression of noisiness is mainly determined by the A-weighted sound pressure level.

The instantaneous judgments sampled every 100 ms are plotted against the values of $L_{\mathrm{eq}}$ of every 100 ms for all the 25 stimuli together in Fig. 3. A fairly high correlation can be found between them. However, there are some deviations from the regression line in some stimuli. These deviations may be due to the difference in the types of helicopter, flight modes, etc.

Examples of the relation between the instantaneous judgments and the values of $L_{\text {eq }}$ of every 100 $\mathrm{ms}$ for each stimulus are shown in Figs. 4 and 5. The stimulus No. 9 shown in Fig. 4 is the noise from the helicopter in taking-off mode and with two blades. High correlation can be seen, but there is a systematic deviation from the regression line. This may be due to the effect of blade-slap noise, which includes a large impulsive component as is shown in Fig. (6)a.
Table 3 Coefficient of correlation between instantaneous judgments and $L_{\mathrm{eq}}$ of every $100 \mathrm{~ms}$.

\begin{tabular}{cc}
\hline Stimulus & $r$ \\
\hline 1 & 0.930 \\
2 & 0.953 \\
3 & 0.954 \\
4 & 0.982 \\
5 & 0.950 \\
6 & 0.958 \\
7 & 0.954 \\
8 & 0.917 \\
9 & 0.950 \\
10 & 0.947 \\
11 & 0.964 \\
12 & 0.982 \\
13 & 0.975 \\
14 & 0.990 \\
15 & 0.968 \\
16 & 0.934 \\
17 & 0.884 \\
18 & 0.944 \\
19 & 0.915 \\
20 & 0.977 \\
21 & 0.951 \\
22 & 0.962 \\
23 & 0.952 \\
24 & 0.969 \\
25 & 0.992 \\
\hline
\end{tabular}

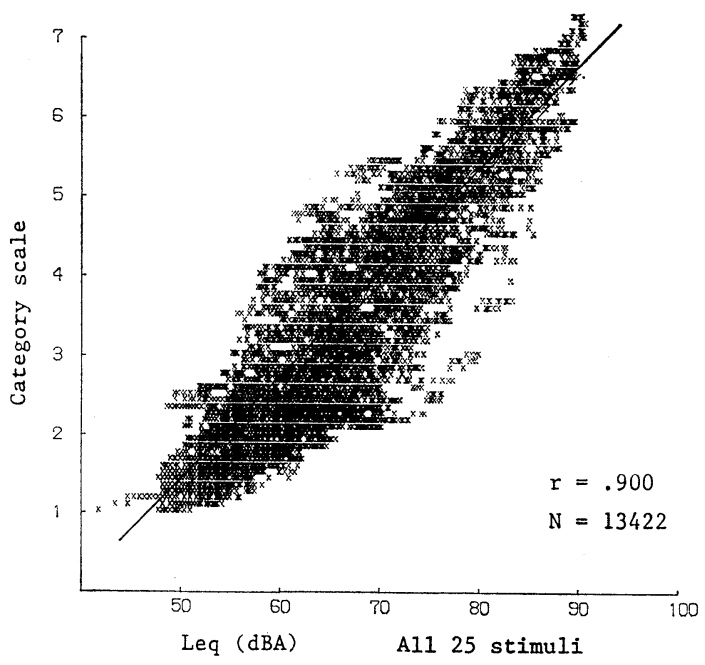

Fig. 3 Relation between the values of $L_{\mathrm{eq}}$ averaged for $100 \mathrm{~ms}$ and the instantaneous judgments of noisiness sampled every $100 \mathrm{~ms}$ for all the 25 stimuli. 


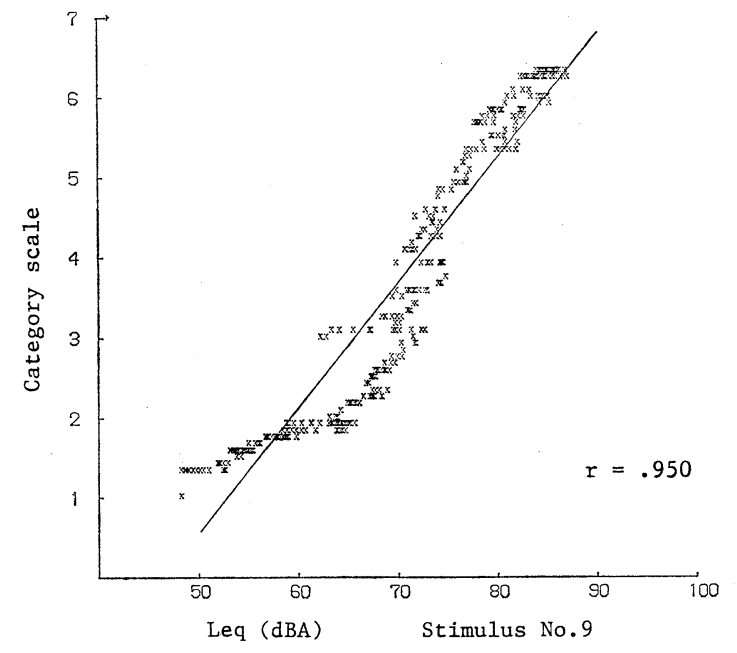

Fig. 4 Relationship between the value of $L_{\text {eq }}$ averaged $100 \mathrm{~ms}$ and instantaneous judgments of noisiness sampled every 100 $\mathrm{ms}$ of stimulus No. 9 which is the noise from the helicopter with two blades and in taking-off mode. A high correlation can be seen between them. However, there can be seen a systematic deviation from the regression line.

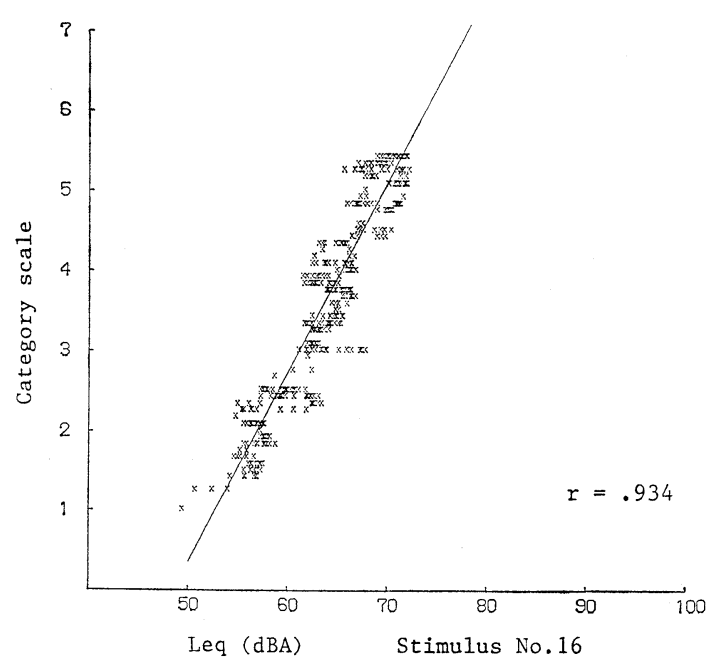

Fig. 5 Relation between the values of $L_{\mathrm{eq}}$ averaged $100 \mathrm{~ms}$ and instantaneous judgments of noisiness sampled every $100 \mathrm{~ms}$ of stimulus No. 16 which is the noise from the helicopter with five blades and in flyover mode. A high correlation can be seen between them and there is no systematic deviations.

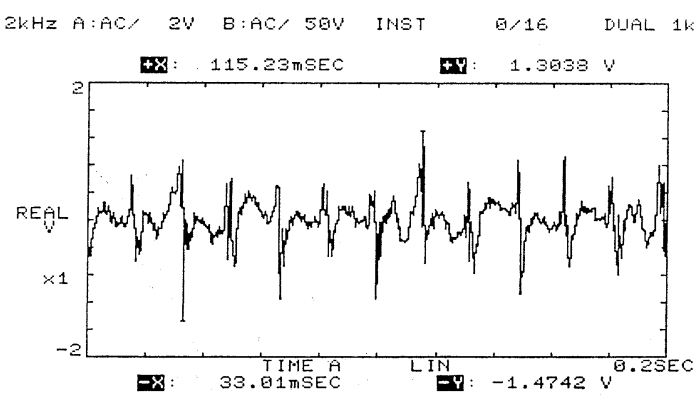

(a)

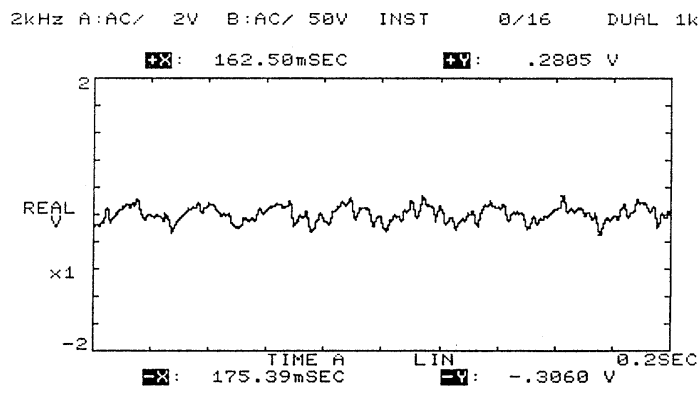

(b)

Fig. 6 Temporal characteristics of helicopter noise. Figure 6(a) shows the analysis of stimulus No. 9 which is the noise from the helicopter with two blades and in taking-off mode. Large impulsive components can be noticed. Figure $6(\mathrm{~b})$ shows the analysis of stimulus No. 16 which is the noise from the helicopter with five blades and in flyover mode.

The temporal variation of the deviations from the regression line is shown in Fig. 7. When a helicopter is approaching, the blade-slap noise is greater. This may cause the overestimation of noisiness. When a helicopter is moving away, the noisiness may be underestimated in contract to the preceding overestimation. The stimulus No. 16 shown in Fig. 5 is the noise from the helicopter in flyover mode and with five blades. In the case of this helicopter, the blade-slap noise is not prominent as is shown in Fig. 6(b), and a good correlation can be seen between the instantaneous judgments and the values of $L_{\mathrm{eq}}$ of every $100 \mathrm{~ms}$.

The blade-slap noise may cause deterioration of 


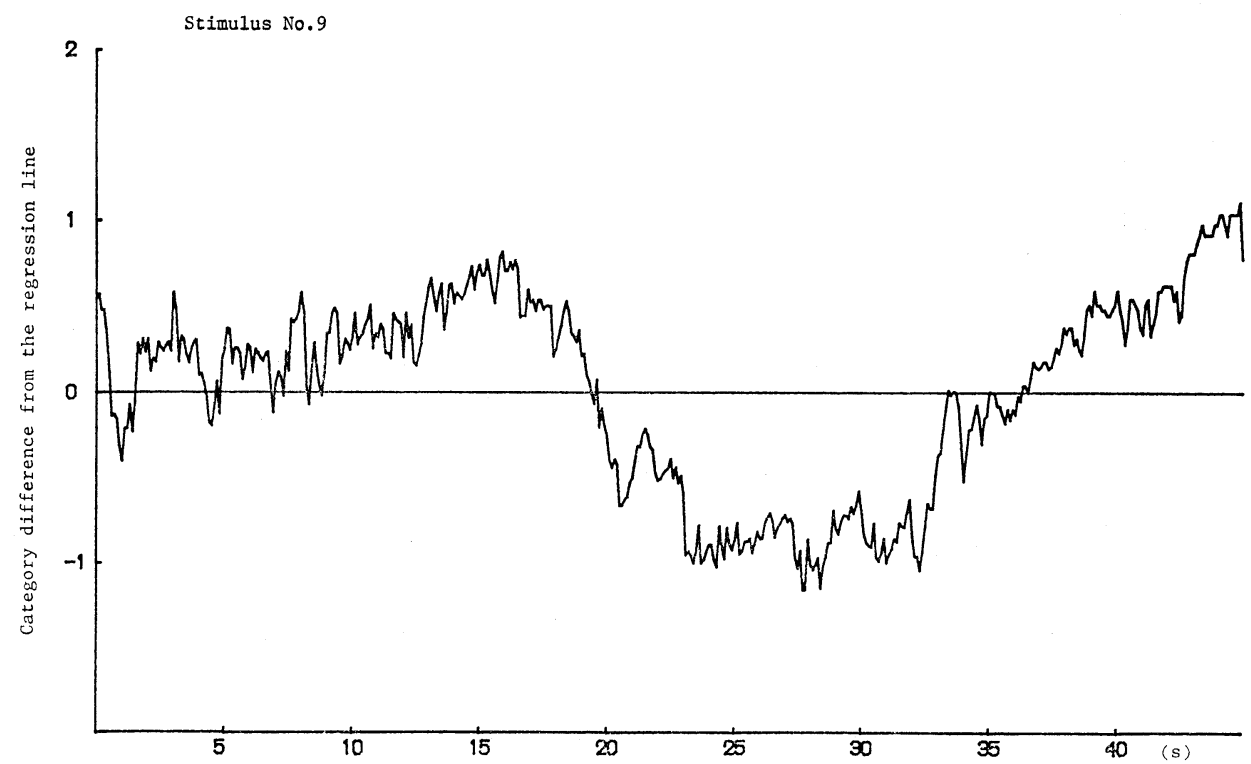

Fig. 7 Temporal variation of the deviations from the regression line for the stimulus No. 9. It can be seen that noisiness was overestimated when the helicopter was approaching and underestimation occurred just after the overestimation.

timbre of helicopter noise. The effect of timbre was examined in Experiments 2 and 3.

\subsection{Instantaneous Impression of Timbre}

The percentages for the selection to each adjective for each five seconds by twelve subjects were calculated. When two or three adjectives were selected in a quick succession, it was dealt that the two or three adjectives were selected continuously. Examples are shown in Figs. 8 and 9. It can be seen that the impression of timbre changes temporally according to the flight pattern and the type of helicopter. The example of the results shown in Fig. 8 is from the same stimulus as shown in Fig. 4, i.e. the noise from the helicopter in taking-off mode and with two blades. When the helicopter was approaching, subjects perceived the noise as being "noisy." Then impression "metallic" became dominant and when it left away the noise was perceived as being "soft." For the stimulus No. 16 from the helicopter in flyover mode and with five blades, the impression of timbre did not change so much during the flight as is shown in Fig. 9. The results of Experiment 2 show that the timbre of some types of helicopter noise varies according to the flight pattern and the flight mode.

\subsection{Overall Judgment}

Examples of semantic profiles averaged for twelve subjects are shown in Figs. 10 and 11. It was found that the overall impression of timbre of the noises were different from each other. Stimulus No. 9 (Fig. 10) gives negative impression: it was perceived as being "loud," "metallic," "gruff," "impure," "annoying," "hard," "unpleasant," "strident," "powerful," and "unpleasing." On the other hand, stimulus No. 16 was judged as being neutral as shown in Fig. 11. The result of factor analysis is shown in Table 4. Factor 1 shows high loading for the adjectives "loud-soft," "gruff-mild," "annoying-not annoying," "strident-calm" and "weakpowerful." Factor 1 can be interpreted as "powerful" factor. Factor 2 shows high loading for the adjectives "metallic-deep," "sharp-dull" and "gentle-hard," and can be regarded as "metallic" factor. Factor 3 shows high loading for the adjectives "pure-impure," "pleasant-unpleasant" and "unpleasing - pleasing," and can be interpreted as "pleasant" factor.

The coefficients of correlation of these adjective scales with $L_{\text {eq }}$ and loudness level based on ISO $532 \mathrm{~B}^{13)}$ (abbreviated as $L L z$ ) are shown in the righthand columns in Table 4. $L L z$ was calculated on the 
Stimulus No.9

(\%)

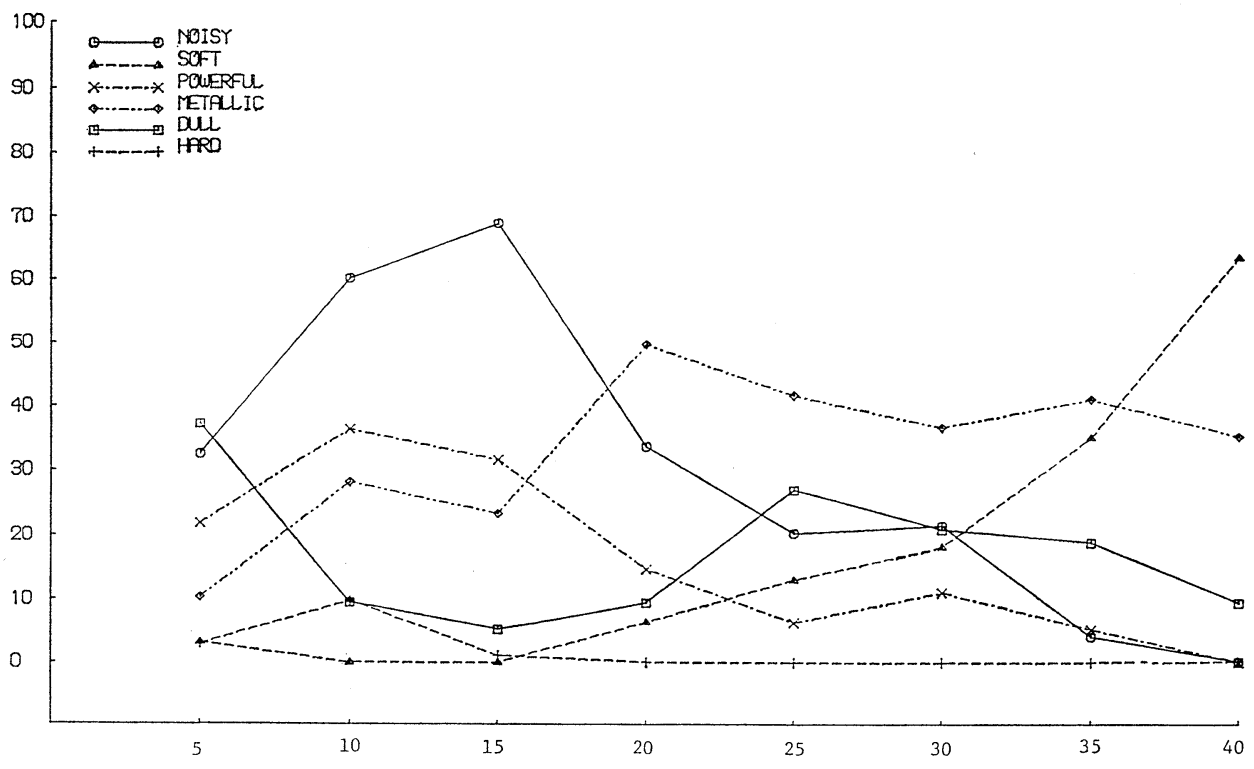

Fig. 8 Temporal change of the timbre of the stimulus No. 9 obtained in Experiment 2. It is noticed that the impression of timbre varied according to the flight pattern.

Stimulus No. 16

(\%)

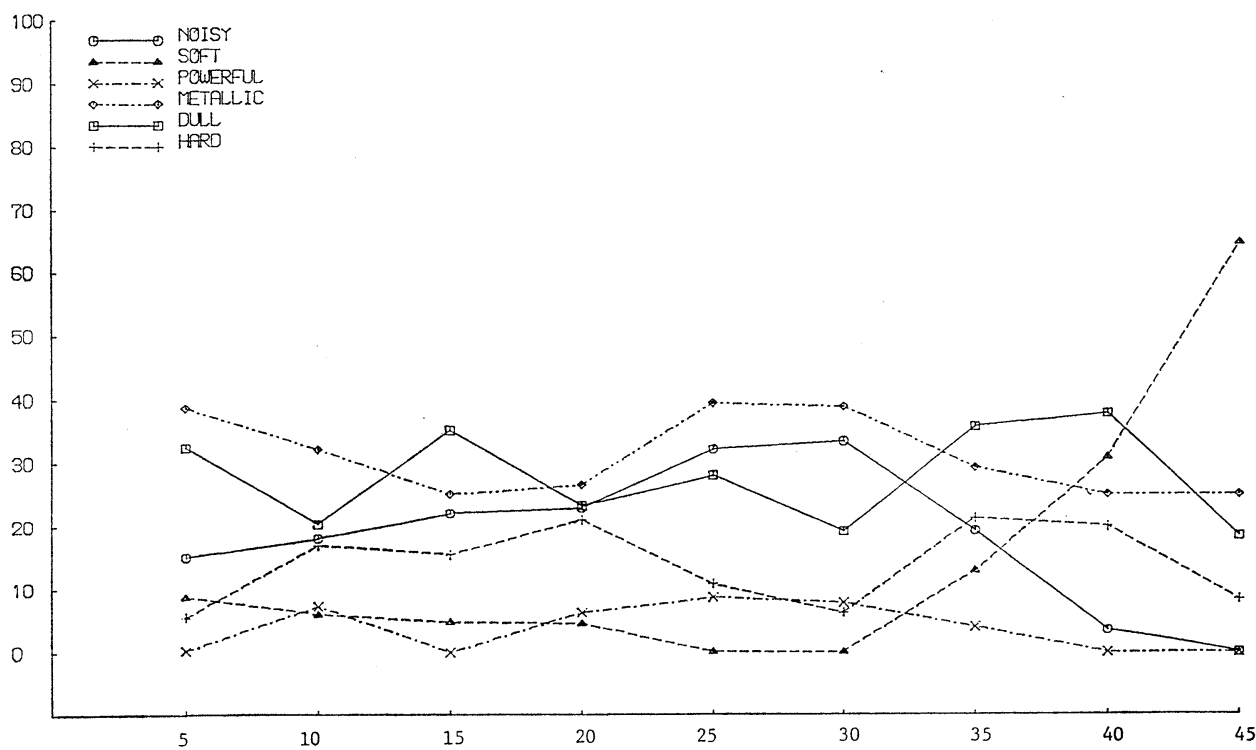

(s)

Fig. 9 Temporal change of the timbre of the stimulus No. 16. Little temporal change can be seen in this case. 


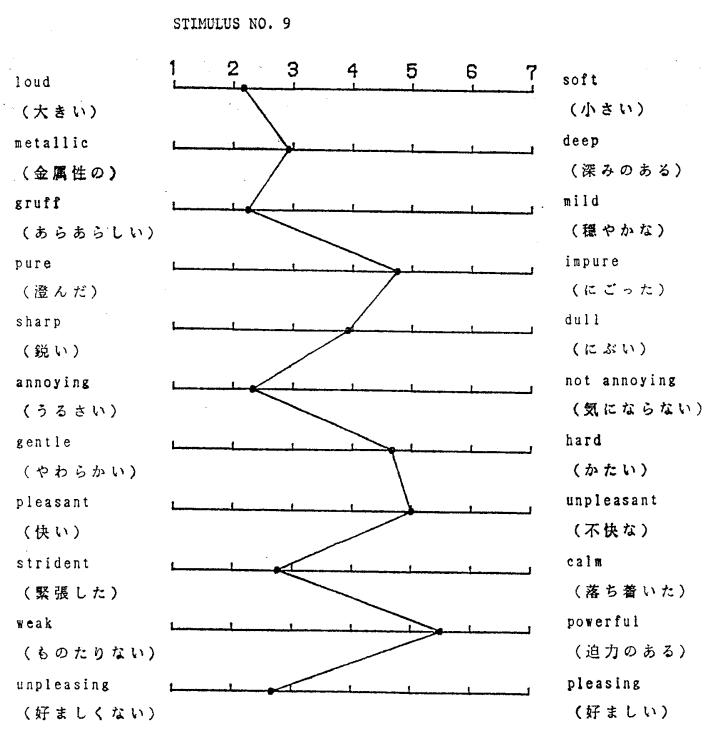

Fig. 10 Semantic profile for the stimulus No. 9 in Experiment 3.

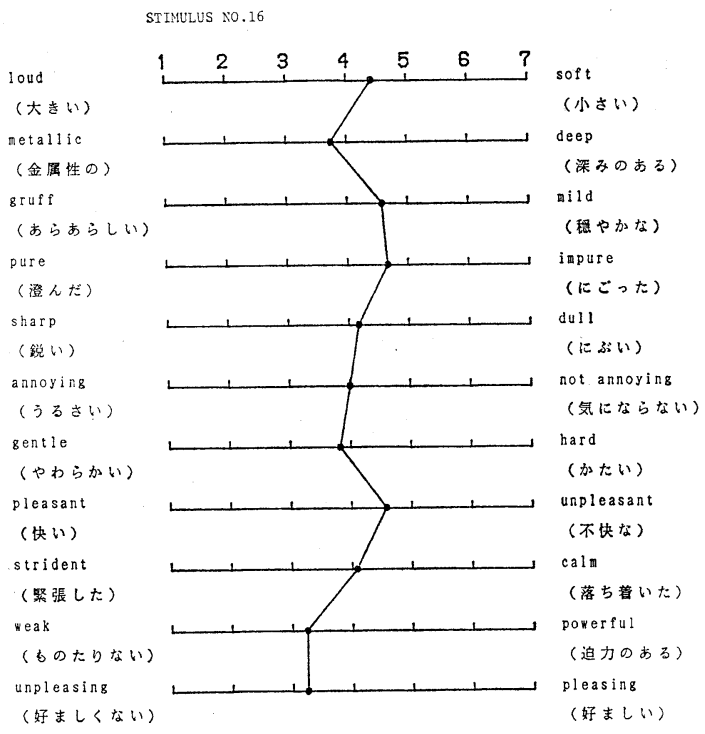

Fig. 11 Semantic profile for the stimulus No. 16.

Table 4 Results of factor analysis.

\begin{tabular}{|c|c|c|c|c|c|c|}
\hline & & I & II & III & $\begin{array}{c}r \\
L_{\mathrm{eq}}\end{array}$ & $\stackrel{r}{L L z}$ \\
\hline 1. loud & - soft & 0.849 & -0.091 & -0.255 & 0.942 & 0.945 \\
\hline 2. metallic & - deep & -0.119 & 0.845 & -0.140 & -0.137 & -0.064 \\
\hline 3. gruff & - mild & 0.737 & 0.170 & -0.381 & 0.947 & 0.950 \\
\hline 4. pure & - impure & -0.098 & 0.152 & 0.741 & -0.696 & -0.676 \\
\hline 5. sharp & — dull & 0.103 & 0.820 & 0.212 & -0.061 & 0.048 \\
\hline 6. annoying & - not annoying & 0.681 & -0.016 & -0.537 & 0.885 & 0.915 \\
\hline 7. gentle & - hard & -0.516 & -0.542 & 0.091 & -0.784 & -0.828 \\
\hline 8. pleasant & - unpleasant & -0.339 & -0.054 & 0.793 & -0.858 & -0.869 \\
\hline 9. strident & - calm & 0.732 & 0.322 & -0.189 & 0.920 & 0.936 \\
\hline 10. weak & - powerful & -0.878 & 0.088 & 0.143 & -0.906 & -0.914 \\
\hline 11. unpleasing & - pleasing & 0.316 & 0.142 & -0.786 & 0.821 & 0.856 \\
\hline
\end{tabular}

basis of the mean energy level of $1 / 3$ octave band. ${ }^{14)}$ Both $L_{\text {eq }}$ and $L L z$ show high coefficient of correlation with the scales of Factor 1 and can be good measures of "powerful" impression of helicopter noise. Neither $L_{\mathrm{eq}}$ nor $L L z$ shows high correlation with the scales of Factor 2. "Metallic" impression can be dominant characteristics for some types of helicopters. In such cases $L L z$ or $L_{\mathrm{eq}}$ is not a good index for the evaluation of helicopter noise. The scales of Factor 3 show correlation with $L_{\text {eq }}$ and $L L z$, though not so high. This suggests that low level sounds tend to be preferred than high level sounds, though other factors also may affect the impressions which belong to Factor 3.

\section{DISCUSSION}

\subsection{Instantaneous Judgments}

In Experiment 1 it was found that instantaneous impressions of noisiness of helicopter noises show a fairly good correlation with $L_{\mathrm{eq}}$ and that they can be approximately evaluated by $L_{\text {eq }}$. However, when they contained strong blade slap noise, there were systematic deviations from the regression lines. Though instantaneous judgments of noisiness showed significantly higher correlation with $L L z$ of every $100 \mathrm{~ms}$ than with $L_{\text {eq }}$, similar deviation was 
found as shown in Fig. 12. This suggests that the overestimation of noisiness may be due to the deterioration of timbre caused by the temporal effect of blade slap noise.

The temporal variations of noisiness (Experiment 1) and timbre (Experiment 2) can be compared, the examples of which are shown in Figs. 7 and 8. It is noticed that the overestimated portions in Experiment 1 correspond to the portions where the adjective "noisy" was selected with high percentages in Experiment 2. This suggests that the dominant impression of timbre of blade slap noise was "noisy." Following underestimated portions in Experiment 1 were judged "dull" or "metallic," etc. in Experiment 2.

\subsection{Comparison between Instantaneous and Over- all Impressions of Timbre}

The percentages with which each adjective was selected in continuous judgment in Experiment 2 were calculated for each stimulus and compared with the category scales in overall judgments in Experiment 3. Examples are shown in Figs. 13 and 14.

A fairly high correlation can be seen between the

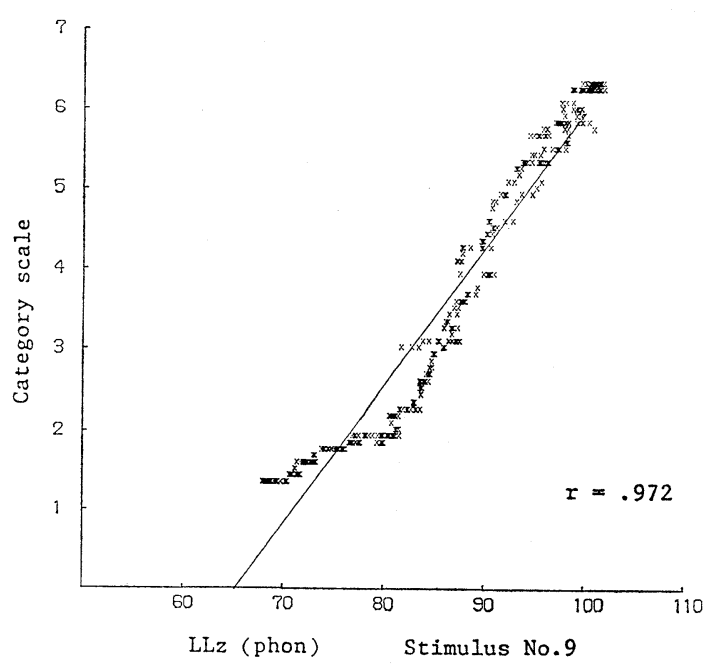

Fig. 12 Relation between $L L z$ averaged 100 ms and instantaneous judgments of noisiness sampled every $100 \mathrm{~ms}$ of stimulus No. 9. Instantaneous judgments showed a higher correlation with $L L z$ than with $L_{\text {eq }}$. However, there still exists a systematic deviation from the regression line. results of continuous judgments and overall judgments in the impression of "powerful" as shown in Figs. 13 (a) and (b). It was found in these figures that noises from the helicopters with two blades and/or in landing mode were judged as being more "powerful" than those with three or five blades, and/or in

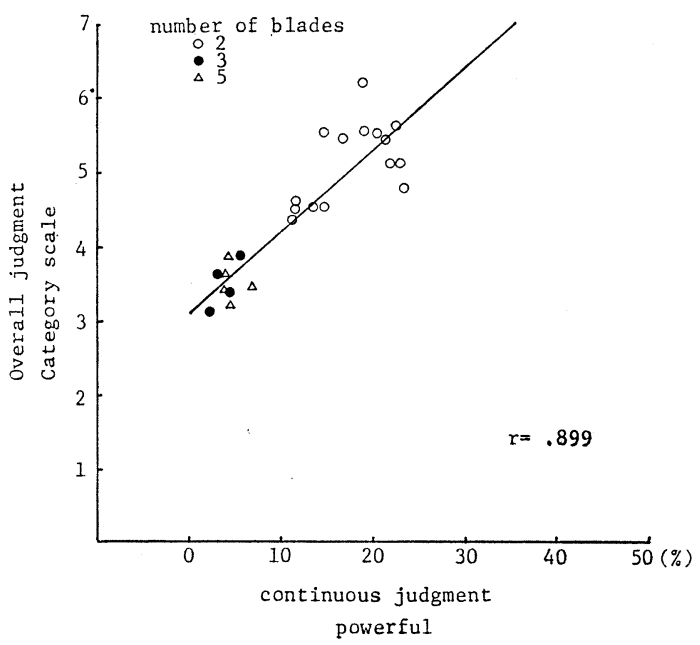

(a)

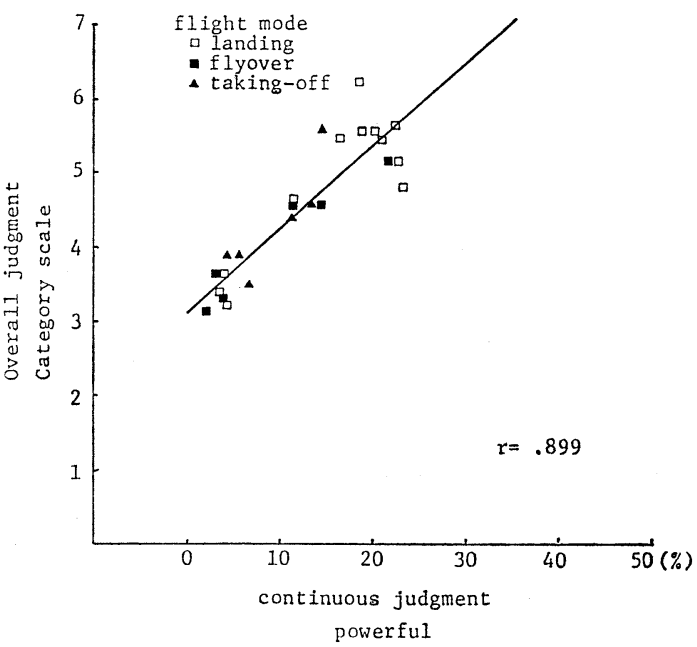

(b)

Fig. 13 Relation between overall judgments and the percentages with which each adjective was selected in continuous judgments for the adjective "powerful." Figure 13(a) shows the effect of the number of blades and Fig. 13(b) that of flight mode. 


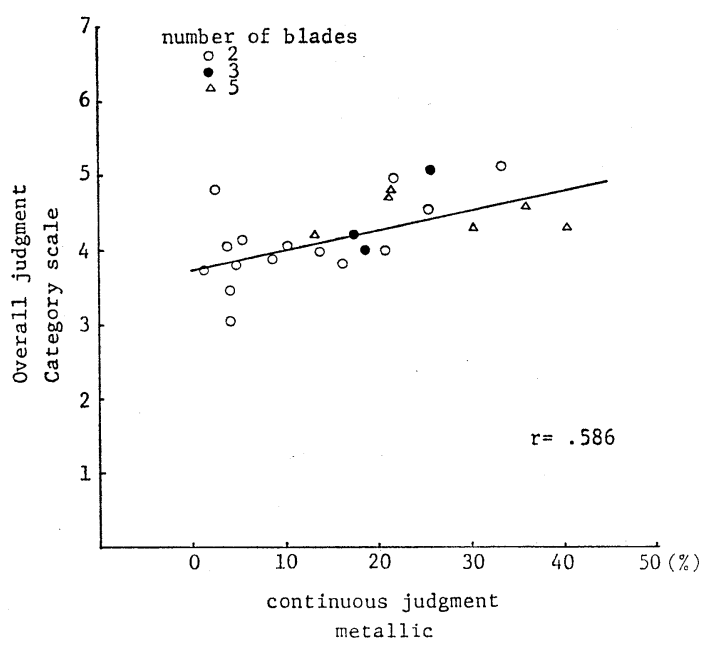

(a)

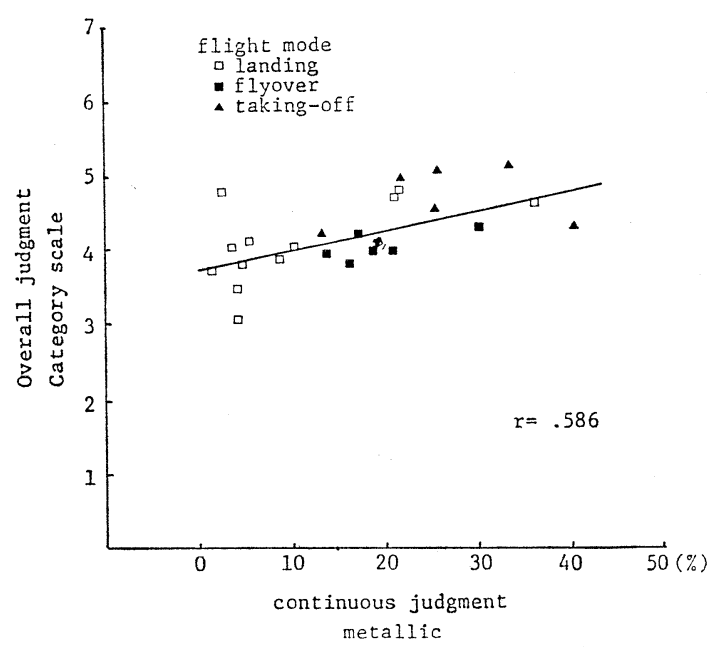

(b)

Fig. 14. Relation between overall judgments and the percentages with which adjective was selected in continuous judgments for the adjective "metallic." Fig. 14(a) shows the effect of the number of blades and Fig. 14(b) that of flight mode.

taking-off or flyover modes.

It was found that the impression of "metallic" was not strong in overall judgments and that the coefficient of correlation is not so high between overall judgments and instantaneous judgments as shown in Figs. 14-a and b. No correlation was found between instantaneous judgments and overall judgments in the impression of "hard" $(r=-0.040)$.
These results suggest that the overall impression is not independent of the instantaneous impressions and that the relation between them is not so simple. The overall impression may be based on the interaction of weighted average of the instantaneous impressions. ${ }^{3)}$ It is also suggested that the impressions of noisiness and timbre are temporally varying and it may be useful to examine the countermeasures of helicopter noises by relating the instantaneous impressions with corresponding physical properties.

\section{FINAL REMARKS}

The method of continuous judgment by category and the method of continuous judgment by selected description were introduced with their procedures and applications as follows.

1. The instantaneous impressions to the sounds which vary along the temporal stream can be measured reliably by these methods.

2. These methods can be applied to everyday sounds, and the relation between stimulus conditions and subjective responses, and the tradeoff effect among stimulus conditions can be examined. This may be helpful to examine the countermeasures for preventing negative impressions of noises.

3. The overall impression of a sound is highly related to the instantaneous impressions. However, the overall impression is not a simple average of the instantaenous impressions. The overall impression may be based on the interaction of weighted average of the instantaneous impressions.

4. Instantaneous impression is affected by the preceding portions of the sounds as well as the corresponding instantaneous sound levels. It was shown how to estimate the time interval which determines the instantaneous impressions.

5. It was discussed that the context of the sounds has an important role in constructing instantaneous impressions.

\section{ACKNOWLEDGMENTS}

The authors are grateful to the members of the Committee of INCE/Japan on Basic Research into Aircraft Noise (chairman: Professor Masaru Koyasu) for offering the recorded helicopter noises used in these experiments and their valuable discussion about the experiments on helicopter noise. 


\section{REFERENCES}

1) S. Namba, T. Nakamura, and S. Kuwano, "An analysis of piano performance," Stud. Hum. Soc. Sci. Coll. Gen. Educ. Osaka Univ. 25, 25-43 (1977) (in Japanese with English abstract).

2) S. Namba and S. Kuwano, "The relation between overall noisiness and instantaneous judgment of noise and the effect of background noise level on noisiness,"’ J. Acoust. Soc. Jpn. (E) 1, 99-106 (1980).

3) S. Kuwano and S. Namba, "Continuous judgment of level-fluctuating sounds and the relationship between overall loudness and instantaneous loudness," Psychol. Res. 47, 27-37 (1985).

4) S. Namba and S. Kuwano, "Psychological evaluation of music performance using the method of continuous judgment by selected description," Harmonia, Kyoto City University for Arts, 18, 633 (1988).

5) S. Namba and S. Kuwano, "Continuous multidimensional assessment of musical performance," J. Acoust. Soc. Jpn. (E) 11, 43-51 (1990).

6) S. Namba, S. Kuwano, T. Hato, and M. Kato, "Assessment of musical performance by using the method of continuous judgment by selected description," Music Percept. 8, 251-276 (1991).

7) R. Teranishi, "Critical rate for identification and information capacity in hearing system," J. Acoust. Soc. Jpn. (J) 33, 136-143 (1977) (in Japanese with
English abstract).

8) S. Namba, T. Yoshikawa, and S. Kuwano, "Effects of range and physical intensity of stimuli on category judgment function," Jpn. Psychol. Res. 10, 95-103 (1968).

9) B. Scharf, "Loudness adaptation," in Hearing Research and Theory, Vol. 2, J. V. Tobias and E. D. Schubert, Eds. (Academic Press, New York, 1983), pp. 1-57.

10) S. Namba, S. Kuwano, and H. Fastl, "Loudneșs of road traffic noise using the method of continuous judgment by category," Proc. Noise 88, 241-246 (1988).

11) S. Kuwano and S. Namba, "Continuous judgment of loudness and annoyance," Proc. 6th Annu. Meet. Int. Soc. Psychophys., 129-134 (1990).

12) S. Namba, S. Kuwano, T. Hashimoto, B. Berglund, D. R. Zhen, A. Schick, H. Hoege, and M. Florentine, "Verbal expression of emotional impression of sound: A cross-cultural study," J. Acoust. Soc. Jpn. (E) 12, 19-30 (1991).

13) ISO 532B, "Acoustics-Method for calculating loudness level" (1975).

14) S. Kuwano, S. Namba, and H. Miura, "Advantages and disadvantages of A-weighted sound pressure level in relation to subjective impression of environmental noises," Noise Control Eng. J. 33, 107-115 (1989). 